International Journal of Current Advanced Research

ISSN: O: 2319-6475, ISSN: P: 2319 - 6505, Impact Factor: SJIF: 5.438

Available Online at www.journalijcar.org

Volume 6; Issue 3; March 2017; Page No. 2530-2533

DOI: http://dx.doi.org/10.24327/ijcar.2017.2533.0043

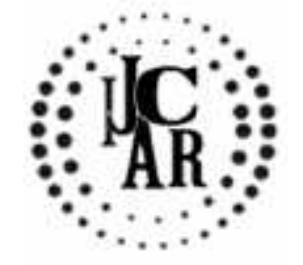

Research Article

\title{
DEVELOPMENT OF CONTROLLER INTERFACE BETWEEN WELDING MACHINE AND ROBOTIC CONTROLLER USING DEVICENET PROTOCOL
}

\author{
Tejashree Balgude* and Satyawan Jagtap
}

Rajarambapu Institute of Technology, Islampur, Distt. Sangli (Maharashtra)

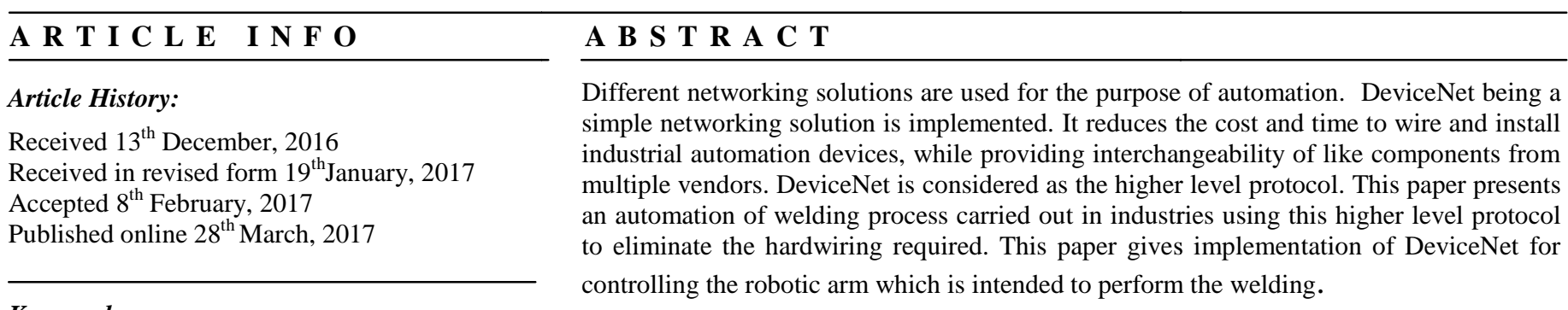

Key words:

CAN,DeviceNet,MODBUS, welding,ODVA,p

ower source.

Copyright $\odot 2017$ Tejashree Balgude and Satyawan Jagtap. This is an open access article distributed under the Creative Commons Attribution

License, which permits unrestricted use, distribution, and reproduction in any medium, provided the original work is properly cited.

\section{INTRODUCTION}

Automation is the new trend in today's world. The automation of different modules is the important aspect in almost every industry. The automation leads to reduced human resources, reduction in the time required in the development of the final product, reduction in the accidents caused during the production process.

The most important advantage of automation is the considerable decrease in the errors caused due to human interference hence more accuracy can be obtained. With the development of technology the automation levels are improving accordingly. The production process includes mode selection, monitoring of different parameters and controlling of those parameters.

Cost of the final product is an important factor concerning its market demand. Automation leads to the reduction in the money required for the production of a particular product which ultimately leads to the overall reduction in the final market price of the product. (1) DeviceNet (2) MODBUS (3) PROFIBUS are the higher level protocols which serves a vital role in most of the industrial automations. These protocols are used as the application layer following the OSI model of networking. This application layer is used over the data link layer which controls the sensors and actuators present in the field. DeviceNet is maintained by ODVA.

*Corresponding author: Tejashree Balgude

Rajarambapu Institute of Technology, Islampur, Distt. Sangli (Maharashtra)
The proposed work intends to develop a protocol which can perform all the controlling actions. The welding parameters like current, voltage are controlled using this communication protocols.

\section{MATERIALS AND METHODS}

This paper describes the most recent developments in the field of conversion between two protocols at all levels of the network stack [Shuo.S, Jianyun.N and Zaiping.C, 2015].

A networked monitoring scheme based on DeviceNet field bus is proposed. The authors Zaiping, Jainfeng and Chao give the way to design the DeviceNet in the explicit message mode by designing the $M$ file [Chao.J, Fan.W, Zaiping.C and Jianfeng.Z, 2009]. It is considered as the strongest mode for design of DeviceNet protocol. A $1747-$ SDN is used as a scanner for the DeviceNet fieldbus. The DeviceNet module is used as slave in the presented work. After the software and hardware was completed the PCI-CAN card interface was used.

Guohong Li, Cheng Xiao and Zhuang Wu in [Cheng.X, Guohong.L and Zhuang.W, 2011] explained the DeviceNet protocol in relevance to its structure. A general purpose microprocessor was described relating it with the DeviceNet protocol. The authors gave a DeviceNet I/O interface development process, communication protocol; object modeling and application layer software design idea. The connection of the host computer with the intelligent devices is the major contribution given by the authors. The host computer is used for high speed of data transmission. 
In order to study the interface of the CAN protocol with the robotic arm for automation [Martinez.H, Morales.A, Martinez.O, 2004] was referred. Authors give the analysis based on the CAN protocol. The components, objects and functions which are required for the robotic arm based automation are described by the authors. The operation of the arm is carried out using the PowerCube modules. Authors preferred the use of the CANopen as the important protocol for helping the communication using the intelligent device.

In [Bonaccorso.F, Bruno.C, Cantelli.C, Longo.D and Muscato.G, 2009] the author has designed an embedded system in order to avoid accidents due to mainly four causes which are glaring at the night time due to incoming vehicle headlights, short circuit faults in the wiring, the engine short circuits due to temperature alarming levels and the last one is the fire outbreak due to the gas leakages. The author has used CAN for communication. He configured the master enabled with gas detection circuit and the slave enabled with Automatic headlight adjustment. He highlights the wide spectrum for application of Controller Area Network.

\section{METHODOLOGY}

The proposed work intends to design a networking system which should perform all the exercises of controlling the parameters by using DeviceNet protocol on the Controller Area Network.

The power supply used is inverter type which is working on $415 \mathrm{~V} \mathrm{ac}, 50 \mathrm{~Hz}$ supply and there is no step down transformer. The main objective behind all this is to implement a digital based communication protocol. Further exploration can be done by creating redundancy at the bus level.

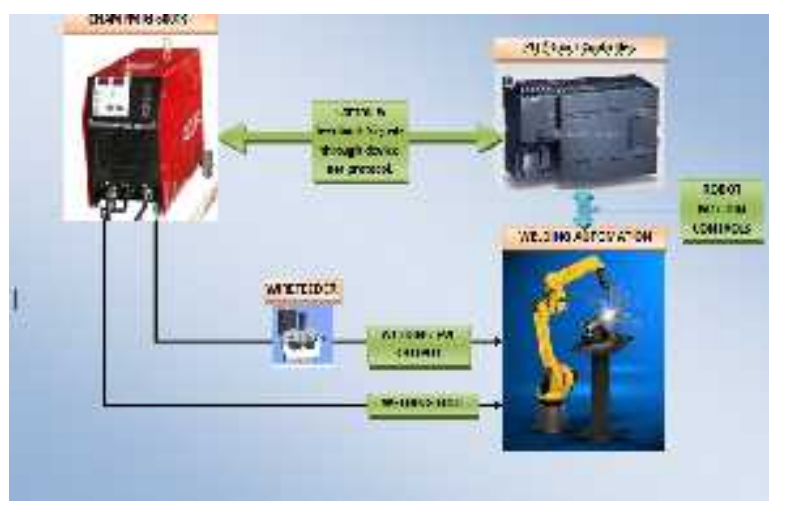

Fig. 1 Block Diagram of Overall System

Project consists of Design \& Development of hardware required for implementation of Device net protocol between welding power source \& Robot for welding automation [4].

The existing system consists of a power supply called CHAMP MULTI 400/600 which is used for the welding of jobs. This power supply is inverter type of supply where a rectifier is used. The PLC of Siemens can be used for the controlling purpose.

\section{A.DeviceNet}

The DeviceNet protocol is a higher level protocol. This is used as the application layer in the proposed application. The controller selected for implementing this protocol uses the Enhanced Controller Area Network (ECAN) module. The other hardware required for this module is the CAN transceiver and a DeviceNet cable which is a coaxial cable consisting of six wires. The CANH and CANL wires are used for the interfacing purpose.

\section{B.MODBUS}

The MODBUS RTU protocol is implemented as an alternative for DeviceNet layer. The RS485 conversion is used for the MODBUS implementation. Same microcontroller as that of DeviceNet is used for MODBUS as well. The same USART module of the controller is used for both the protocols. The half duplex method of communication is used for the MODBUS application. The input for the buffer controller board is from the power supply used for welding. The power supplies used for welding in the industry is called as CHAMP MIG.

\section{Hardware Implementation}

The hardware used basically includes a control PCB which acts as a controlling unit that provides feedback. These feedback signals are analyzed and appropriate controlling action is performed accordingly. The microcontroller IC used for the application is MICROCHIP'S PIC18F4580. Other hardware requirement includes a CAN transceiver IC MCP2561. MAX232 is used as the RS232 driver IC which converts the TTL level into RS232 logic level. The MAX490 IC from MAXIM is used for the RS485 conversion which is required for the MODBUS implementation.

The FOD817 is used as the isolator. A DeviceNet gateway from BECKOFF is used for the interfacing of the circuit board with the robotic arm. The KRC2 robotic arm at KUKA robotics is to be used for demonstration purpose. The description of important modules and their relevant functioning in the circuit is given below.

\section{Microcontroller}

- PIC18F4580 is used as the microcontroller.

- It includes the ECAN module which supports DeviceNet application.

- It includes Enhanced Universal Synchronous Asynchronous Receiver Transmitter (EUSART) and the EUSART module supports both full duplex as well as half duplex communication.

\section{CAN Transceiver}

CAN acts as the basic layer for the DeviceNet protocol. The CAN transceiver is used so as to establish the interface between the two layers of the protocol stack. CANH and CANL wires of the transceiver are connected to the gateway. It supports $12 \mathrm{~V}$ or $24 \mathrm{~V}$ systems. The speed of $1 \mathrm{Mbps}$ can be achieved using this IC.

\section{RS232 and RS485 Drivers}

The conversion of different voltage levels is required for carrying out the communication. The TIA/EIA-232 levels are converted to TTL levels using the MAX232. Similarly the MAX490 is used for the TIA/EIA-485 level conversion.

\section{Gateway}

- The gateway provided by the BECKOFF is used.

- It uses $24 \mathrm{~V}$ supply for operating.

- A gateway can convert one protocol type to another. This application implements the conversion of EtherCAT to DeviceNet. 
- It comprises of a 6 pins connector. The pin configuration starting from the first pin is $+\mathrm{V}$, CANH, SHIELD, CANL, -V respectively.

- A DeviceNet cable which is a six wire coaxial cable is connected to this connector of the gateway. A Seimens PLC is used for the controlling of the signals.

- Basic signals are obtained from the power supply. Compatibility is required for controlled actions.

\section{Software Implementation}

The software design includes implementing of a stack which is based on the layers structured. Each layer implements a corresponding module of the software. The basic datalink layer that uses the CAN module implements software which performs the communication between two boards. The application layer uses the common industrial protocol (CIP) specifications and the network adaptations of CIP for DeviceNet. For the implementation of the configuration file, 'TwinCAT Manager' is used which is required by the gateway.This gateway.

DeviceNet Software: The DeviceNet software includes the following elements

EDS file: It is an Electronic Configuration file which can be generated using the configurator tool. Parameters are set in this electronic file according to the requirement.

Explicit Messaging: Messages are to be transmitted and received and the controlling action is to be performed if an error occurs. This type of messaging defines the location of the receiving end which is also called a client.

\section{Software Design}

Software design consists of different steps which are summarized as follows

- Initialization: The initialization consists of two processes first is the initialization of the controller and next is the initialization of the CAN module.

- Message Transmission: For the DeviceNet module the message transmission is done through CAN bus. The power supply transmits the control signals to the first node of the CAN bus. The USART messages are converted into the CAN messages.

- Message Reception: For the DeviceNet module the reception of the CAN message is done by the second node on the CAN bus.

The stack implementation includes adding the class, attributes, instances and objects required for DeviceNet. An EDS file given by the manufacturer of the gateway device which is being used is required. This EDS file is to be imported in the software where the configuration of the overall communication link is designed.

Table 1 String Format for communication

\begin{tabular}{|c|c|}
\hline $\begin{array}{l}\text { String From power } \\
\text { Source to Remote }\end{array}$ & Bytes \\
\hline Start Pointer & $\$$ \\
\hline Hi_actual current & 0x00 1byte \\
\hline Lo_actual current & 0x00 2byte \\
\hline Hi_actual voltage & 0x00 3 byte \\
\hline Lo_actualvoltage & 0x00 4 byte \\
\hline Psstatusbyte & 0x00 5 byte \\
\hline
\end{tabular}

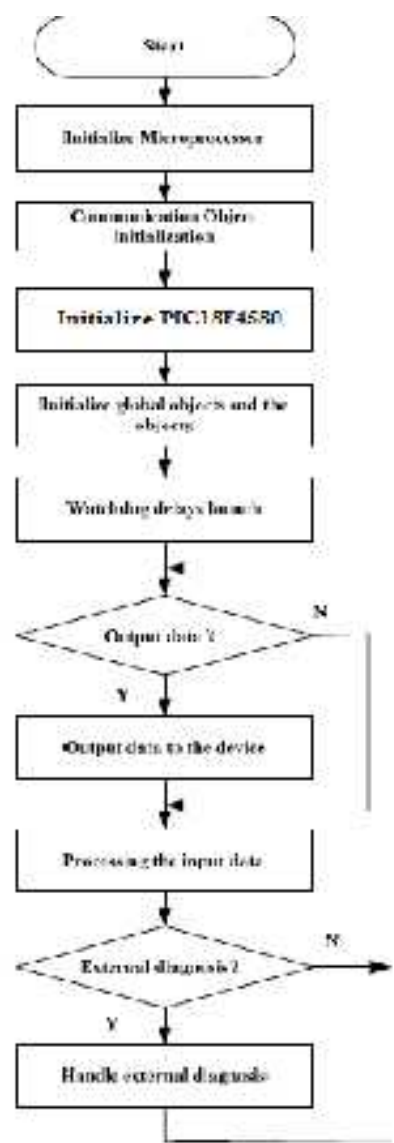

Fig. 2 Flowchart of software implementation.

Table shows the string format which is used to assign the bytes for the controlling signals. Parameters like current, voltage, wirefeeder length are controlled using this bytes.

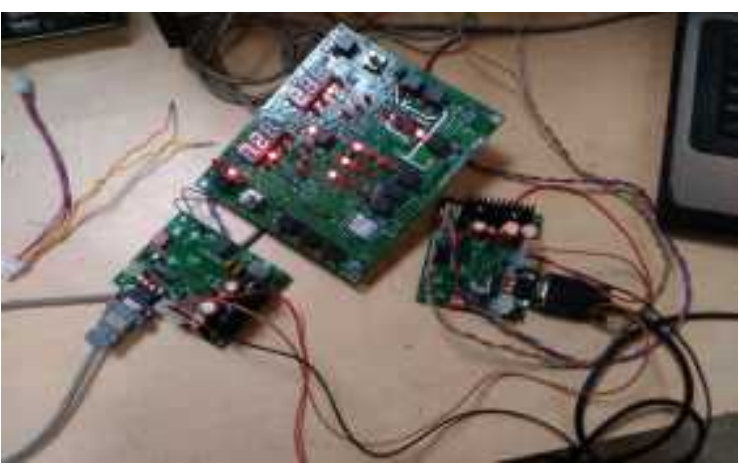

Fig 3 Hardware setup

The hardware setup is shown in figure 5. The bigger board is the power supply PCB while the smaller two PCBs are the CAN communication PCBs. The setup is connected to the personal computer through USB to serial cable.

\section{CONCLUSION}

The hardware and software required for implementing the protocol are being developed and the welding power supply will be interfaced with the controlling PCB to carry out the welding operation. Thus the hardwiring required will be eliminated adversely reducing the cost and time required to perform welding. 


\section{RESULTS}

The results obtained are for one module that is the CAN module. Also the results for the datalink layer required for the protocol along with its physical layer are obtained.

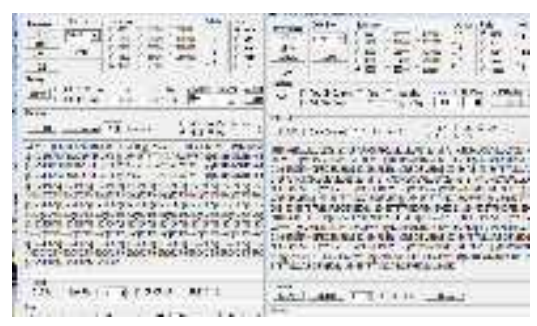

Fig. 4 Communication between two nodes

The terminalv1.9b by Br@y++ software is used for checking the reception of the message. The digital are to be received at CANTX and CANRX pins which conforms that the communication link is established between the transmitter and receiver. A string format is followed for the reception of the data.

The signals obtained are checked on the digital oscilloscope to determine the recessive and dominant bits obtained.

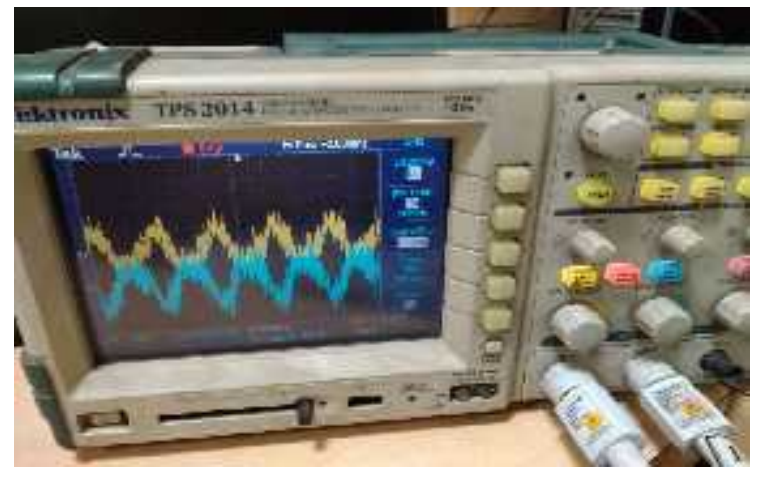

Fig $5 \mathrm{CANH}$ and CANL signals

The data is transformed in bits form where each bit is assigned certain value. A message that is 'STRINGRECEIVED' is obtained when the correct number of bytes is being sent from the transmitter side.

\section{Acknowledgment}

The project is sponsored by 'ADOR Welding Limited, Pune'. I would thank all the employees, Team leader and Head of The Department at the sponsor company. The contribution of the faculty and my guide at Rajarambapu Institute of Technology was of immense for the proposed work

\section{References}

1. Shuo.S, Jianyun.N and Zaiping.C, 2015 The Design of a DeviceNet - SPI Converter Module Based on the STM32 MCU, IEEE Transactions Intelligent Transportation System, vol. 16, No 2.

2. Chao.J, Fan.W, Zaiping.C and Jianfeng.Z, 2009, Power Monitor Networked Control Based on DeviceNet Field-bus, IEEE Transactions Intelligent Transportation System, 18-19.

3. Cheng.X, Guohong.L and Zhuang.W, 2011, Development and Application Control Network Based on DeviceNet, IEEE.

4. Martinez.H, Morales.A, Martinez.O, 2004, Automation of Robotic Arm by analysing the CAN protocol, IEEE, Proceedings of the $14^{\text {th }}$ International Conference on Electronics, Communication and Computers.

5. Bonaccorso.F, Bruno.C, Cantelli.C, Longo.D and Muscato.G, 2009, A Modular Software Inerface for the Control System of an Arc Welding Robot, IEEE.

6. Dawei.Y, Kui.L, Jinli.Y and Guanying.Z, 2008, Residual Current Monitoring Based on DeviceNet, Proceedings of the $7^{\text {th }}$ World Congress On Intelligent Control and Automation, IEEE,.

7. Gurrala.S, 2015, Implementation of CAN bus and RS232/485 Protocol Conversion Interface, International Journal of Scientific Engineering and Technology Research, vol. 04.

8. Junhao.N, 2012, Design of USB-CAN controller based on PIC18F4580, International workshop on Information and Electronics Engineering(IWIEE).

9. Wang.X and Guo.W, 2009, The Design of RS232 and CAN Protocol Converter based on PIC MCU, Computer and Information Science, August 2009.

\section{How to cite this article:}

Tejashree Balgude and Satyawan Jagtap (2017) ' Development of controller interface between welding machine and robotic controller using devicenet protocol', International Journal of Current Advanced Research, 06(03), pp. $2530-2533$. DOI: http://dx.doi.org/10.24327/ijcar.2017. 2533.0043 